\title{
Disrupted fibroblastic reticular cells and interleukin-7 expression in tumor draining lymph nodes
}

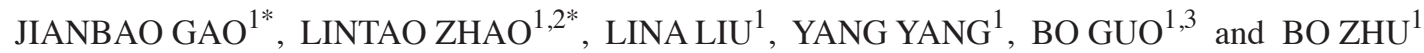 \\ ${ }^{1}$ Department of Oncology, Xinqiao Hospital, Third Military Medical University, Chongqing 400037; \\ ${ }^{2}$ Department of Oncology, The People's Liberation Army No. 324 Hospital, Chongqing 404000; \\ ${ }^{3}$ Department of Pathogenic Biology, Third Military Medical University, Chongqing 400038, P.R. China
}

Received February 8, 2016; Accepted April 28, 2017

DOI: 10.3892/ol.2017.6537

\begin{abstract}
The immune system of patients with cancer is usually in an inhibitory state. Lymph node (LN) draining of pathological sites provides a suitable microenvironment where adaptive immune responses mainly occur. However, the microenvironment in the tumor draining lymph nodes (TDLNs) of patients with cancer appears to be in favor of tolerance. The effects of tumor cells on TDLNs have not been elaborated clearly. The present results have indicated that tumor cells may directly affect TDLNs by decreasing the fibroblastic reticular cell population that led to less interleukin-7 secretion. As a result, the number of T cells in TDLNs declined with reduced survival signals. A decreased number of $\mathrm{T}$ cells in TDLNs means weakened ability of immune surveillance. Clinically, these results were also confirmed in LN biopsies from patients with colon cancer at different clinical stages. Results of the present study showed that tumor cells may directly inhibit the immunological function of TDLNs.
\end{abstract}

\section{Introduction}

The immune system of patients with cancer is often in an inhibitory state, and is unable to fulfill responsibilities of

Correspondence to: Professor Bo Guo, Department of Pathogenic Biology, Third Military Medical University, 30 Gaotanyan Street, Chongqing 400038, P.R. China

E-mail: guobomail@gmail.com

Professor Bo Zhu, Department of Oncology, Xinqiao Hospital, Third Military Medical University, 30 Gaotanyan Street, Chongqing 400037, P.R. China

E-mail: xinqiaooncology@126.com; bo.zhu@tmmu.edu.cn

*Contributed equally

Abbreviations: FRC, fibroblastic reticular cells; LNs, lymph nodes; TDLNs, tumor draining lymph nodes

Key words: cancer, interleukin-7, tumor draining lymph node, fibroblastic reticular cells immunological surveillance (1). These patients often suffer from decreased $\mathrm{T}$ cell counts, which lowers their ability to cope with different pathogens (2). A large number of immunosuppressive cells accumulate in tumor microenvironments, including exhausted cytotoxic $\mathrm{T}$ lymphocytes, Tregs and myeloid derived suppression cells, which limits the normal function of effector immune cells (3).

Generation of adaptive immune responses mainly takes place in secondary lymphoid organs, particularly lymph node (LN) draining of pathological sites. Antigen-specific lymphocytes are predominantly activated and expanded in draining LNs (4). However, the microenvironment in tumor draining lymph nodes (TDLNs) of patients with cancer appears to be in favor of immune tolerance (5). Cell components of LNs consist of immune cells and stromal cells. According to their different surface markers [CD31 and podoplanin (gp38)] stromal cells can be separated into several subtypes (6). Fibroblastic reticular cells (FRCs; gp $\left.38^{+} \mathrm{CD} 31^{-}\right)$are the main constructer in LNs, which orchestrate a complex network of extracellular matrix and collagens (4). Lymphocytes then roam through this network to search for cognate antigens expressed on the surfaces of antigen-presenting cells (7). This interaction between stromal cells and immune cells in LNs is critical for the normal function of the immune system (7-9). Simultaneously, FRCs are the main source of interleukin-7 (IL-7) in the periphery, a survival signal to lymphocytes immigrated into LNs (10). Competition for this limited signal may control the size of the T lymphocyte pool (11). Lymphatic endothelial cells $\left(\mathrm{gp} 38^{+} \mathrm{CD} 31^{+}\right)$circle around the lymphatic vessel in LNs and are another producer of IL-7 in mice and human fetal mesenteric LNs (12). These stromal cells are candidate compartments for the maintenance of peripheral immune tolerance (6,13-15).

Accordingly, the present study aimed to elucidate whether tumor cells have an impact on LN stromal cells, to deteriorate their immunosuppressive status in patients with cancer. The present study focused on IL-7 produced by stromal cells, a cytokine indispensable for the adaptive immune system (16). $\mathrm{T}$ cell homeostasis and lymphopenia-induced proliferation are highly dependent on peripheral IL-7 $(17,18)$, and are also crucial for T lymphopoiesis in the thymus (19) and LN organogenesis (20-22). The present study identified that IL-7 produced by FRCs in TDLNs of tumor-bearing mice was significantly decreased. Inadequate supply of IL-7 in TDLNs 
was insufficient to support the survival of T cells in LNs, which will aggravate the immunosuppressive status of cancer.

\section{Materials and methods}

Mice. Approval for animal experiments was obtained from the Institutional Ethics Committee of the Third Military Medical University (TMMU; Chongqing, China). Female 6-week-old C57BL/6 mice, weighting 16-18 g, came from the Center of Experimental Animals of TMMU. They were housed in SPF-class laboratory animal room at a temperature of $22-26^{\circ} \mathrm{C}$ and humidity of $50-60 \%$. They were maintained under a $12 \mathrm{~h}$ dark $/ 12 \mathrm{~h}$ light cycle, supplied with sterile food and water ad libitum. All animals were assigned to control and tumor-bearing groups randomly. A total of $1 \times 10^{6}$ Lewis lung carcinoma cells, suspended in $100 \mu$ l PBS buffer, were inoculated in the right inguinal region of mice.

Patient cohorts. A total of 40 patients diagnosed with colon cancer at different Dukes stages (23) who had received surgical excision in Xinqiao Hospital were included in the present study. Ethical approval for the project was obtained from the Institutional Ethics Committee of the TMMU. Written informed consent was obtained from each patient included in the present study.

LN biopsy specimens. A total of 40 patients with colon cancer at different Dukes stages were enrolled into the present study. LN biopsies, from patients undergoing surgical resection or colonoscopic biopsy were obtained from the Department of Pathology, Xinqiao Hospital (Chongqing, China) randomly. All LN biopsies came from mesenteric LNs which were defined as TDLNs. According to the criteria of Dukes stages (23), each stage (A-D) contained 10 patients, and there were 1 to 4 LNs for each patient. The study mainly focused on the changes of IL-7, number of T cells and FRCs in TDLNs. As a result, TDLNs from Dukes stage A were used as control groups in the patient experiment. Mice were sacrificed 28 days following tumor inoculation, and subsequently the LNs of the mice were removed from the inguinal region. The right LNs of the inguinal region were defined as TDLNs while the left sides were defined as non-TDLNs (nTDLNs). LNs from control group mice were defined as control specimens (Con). Each LN biopsy was immediately immersed in $4 \%$ neutral buffered paraformaldehyde and paraffin embedded.

Flow cytometry. Flow cytometry analysis was performed as previously described (24). LNs were dissected and digested thoroughly with enzyme mix comprised of RMPI-1640, containing $1 \mathrm{mg} / \mathrm{ml}$ collagenase IV (Sigma-Aldrich; Merck KGaA, Darmstadt, Germany), $2 \%$ fetal bovine serum (Tianjin TBD standard, Tianjin, China) and $50 \mu \mathrm{g} / \mathrm{ml}$ DNase I (Invitrogen; Thermo Fisher Scientific, Inc., Waltham, MA, USA). The following antibodies were used: Anti-CD45 (clone 30-F11); anti-podoplanin (clone8.1.1); anti-CD31 (cloneMEC13.3); anti-CD4 (clone GK1.5); and anti-CD8 (clone 53-6.7; all from BioLegend, Inc., San Diego, CA, USA). All the dilutions were 1:100.

Reverse transcription-quantitative polymerase chain reaction (RT-qPCR). Total RNA was extracted using TRIzol reagent
(Invitrogen; Thermo Fisher Scientific, Inc.), according to the manufacturer's protocol. RNA was converted to complemenatry (c)DNA using PrimeScript Reverse Transcription reagent kit with gDNA eraser (Takara Bio Inc., Otsu, Japan). The cDNA from was used for RT-qPCR analysis of IL-7. PCR primer pairs were as follows: IL-7 forward, 5'-CTGCAGTCC CAG TCATCAGTA-3' and reverse, 5'-GTGGCACTCAGA TGATGTGACA-3' and $\beta$-actin forward, 5'-CCTGAGGCT CTTTTCCAGCC-3' and reverse, 5'-AGAGGTCTTTACGGA TGTCAACGT-3' (25). Total IL-7 mRNA was measured using SYBR-Green reagents (Takara Bio Inc.) and run in triplicate on the Applied Biosystems 7500 fast real-time PCR Detection system (Thermo Fisher Scientific, Inc. Carlsbad, CA, USA), normalized to $\beta$-actin by employing the $2^{-\Delta \Delta C q}$ method (26). Thermocycling conditions were as follows: $95^{\circ} \mathrm{C}$ for $30 \mathrm{sec}$, followed by 40 cycles of $95^{\circ} \mathrm{C}$ for $5 \mathrm{sec}, 59^{\circ} \mathrm{C}$ for $34 \mathrm{sec}, 95^{\circ} \mathrm{C}$ for $15 \mathrm{sec}, 60^{\circ} \mathrm{C}$ for $60 \mathrm{sec}$ and $95^{\circ} \mathrm{C}$ for $15 \mathrm{sec}$.

Immunofluorescence microscopy. All staining was performed as previously described using $10 \mu \mathrm{m}$ tissue sections mounted on glass slides $(8,9)$. Primary antibodies were as follows: Anti-mouse ER-TR7 (dilution, 1:100; cat. no. sc-73355; Santa Cruz Biotechnology, Inc., Dallas, TX, USA); anti-mouse IL-7 (dilution, 1:50; cat. no. sc-7921; Santa Cruz Biotechnology Inc.); and rabbit IgG isotype (cat. no. A7016; Beyotime Institute of Biotechnology, Beijing, China). Secondary antibodies were fluorescein isothiocyanate-conjugated anti-rat (dilution, 1:200; cat. no. A0557; Beyotime Institute of Biotechnology) and $\mathrm{Cy} 3$-conjugated anti-rabbit $\operatorname{IgG}$ (dilution, 1:100; cat. no. A0516; Beyotime Institute of Biotechnology).

Western blot analysis. Portions of each LN sample were applied to $12 \%$ SDS-PAGE and detected using a specific goat gp38 antibody (dilution, 1:1,000; cat. no. AF3244; R\&D Systems, Inc., Minneapolis, MN, USA). A horseradish peroxidase-conjugated anti-goat $\operatorname{IgG}$ (dilution, 1:5,000; cat. no. A0181; Beyotime Institute of Biotechnology) was used to visualize the signal by ECL prime western blotting (Beyotime Institute of Biotechnology). The blots were quantified by measuring the relative band intensity normalized to changes in $\beta$-actin intensity.

Immunohistochemistry. All staining procedures were performed as previously described (27). Subsequent to tissues being deparaffinized and rehydrated using dimethylbenzene and gradient concentration of ethanol, heat-induced epitope retrieval was performed with water bath heating in EDTA antigen retrieval buffers ( $\mathrm{pH}$ 8.0; Zhongshan Company, Beijing, China) for $10 \mathrm{~min}$, cooling to room temperature. The sections were then blocked with $5 \%$ bovine serum albumin (BSA) blocking reagent (Boster Systems, Inc., Pleasanton, CA, USA) and $3 \% \mathrm{H}_{2} \mathrm{O}_{2}$. Primary antibodies were diluted in 5\% BSA and incubated overnight at $4^{\circ} \mathrm{C}$. The next day, slides were washed using PBS and stained with secondary antibody, horseradish peroxidase-conjugated anti-rabbit (dilution, 1:1,000; cat. no. A0208; Beyotime Institute of Biotechnology). Light micrographs were captured using an Olympus BX60 upright microscope (magnification, $x 40 ; 0.75$ numerical aperture).

Primary antibodies were as follows: Anti-mouse CD3 (dilution, 1:100; cat. no. ab16669; Abcam, Cambridge, MA, 
A

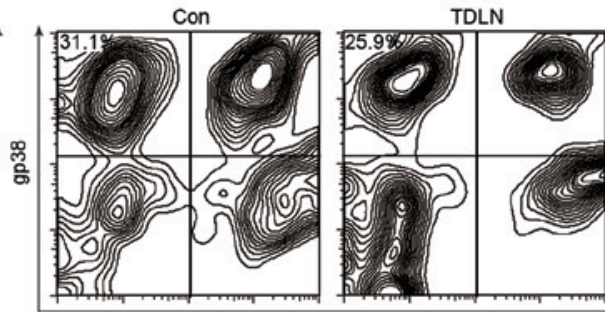

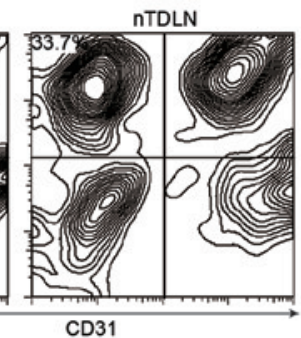

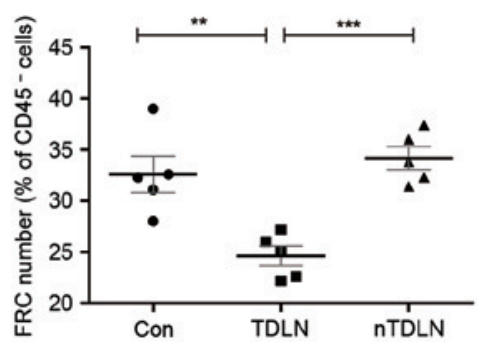

B
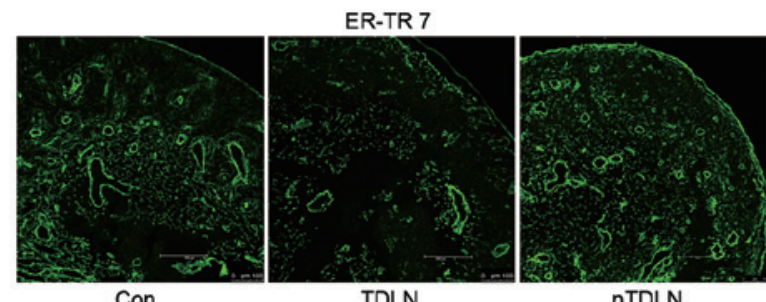

nTDLN

C

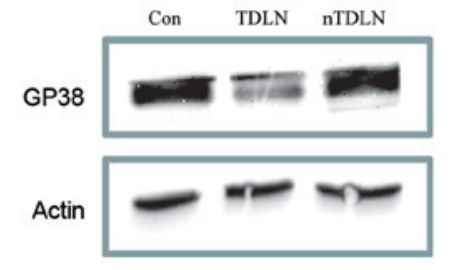

D

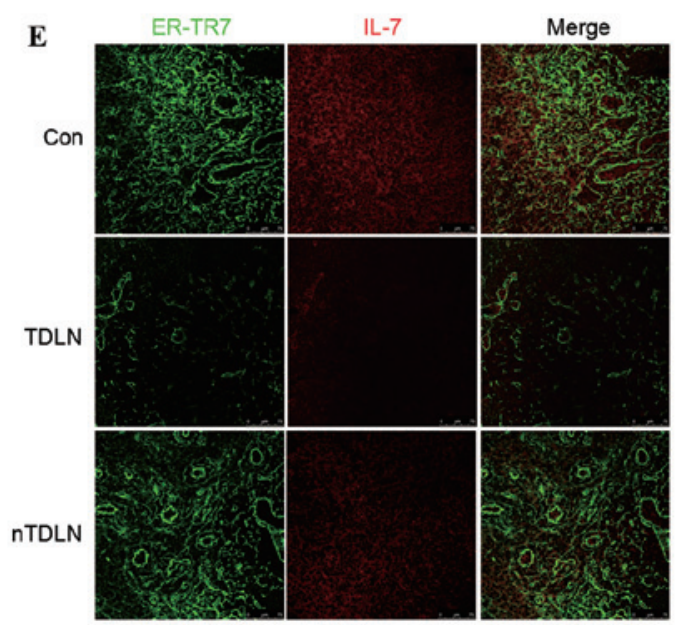

Figure 1. FRCs and IL-7 were significantly decreased in TDLNs. (A) Flow cytometric analysis showed that the number of FRCs (gp38 $\left.{ }^{+} \mathrm{CD} 31^{-}\right)$in $\mathrm{CD}^{-} 5^{-}$cells dropped in TDLNs, representative of 5 mice for the control group and 5 mice for the tumor-bearing group. (B) Immunofluorescent staining showed lower expression of ER-TR7 in TDLNs. (C) Gp38 detected by western blot analysis normalized to changes in the $\beta$-actin intensity. (D) The estimated amounts of IL-7 mRNA were calculated with the reverse transcription-quantitative polymerase chain reaction. IL-7 expression in TDLNs significantly decreased compared with the control group. The mean values \pm standard error of the mean were analyzed from 4 mice per group, in 3 independent experiments respectively. (E) Confocal images of LNs sections stained for ER-TR7 (green) and IL-7 (red). ${ }^{* *} \mathrm{P}<0.01,{ }^{* * *} \mathrm{P}<0.001$. IL-7, interleukin-7; LN, lymph node; TDLN, tumor draining lymph node; FRC, fibroblastic reticular cell.

USA); anti-mouse CD8 (dilution, 1:100; cat. no. sc-7188; Santa Cruz Biotechnology Inc.); anti-human IL-7 (dilution, 1:50; cat. no. sc-7921; Santa Cruz Biotechnology Inc.); rabbit IgG isotype (cat. no. A7016; Beyotime Institute of Biotechnology); anti-human CD8 (cat. no. ZA-0508; Zhongshan Company, Beijing, China); and anti-human desmin (cat. no. ZA-0610; Zhongshan Company). Quantitative image analysis was performed using 5-10 randomly acquired, high-powered images (magnification, $\mathrm{x} 400$ ). The number of cells in each image was manually counted and the percentage of IL-7 and desmin area was calculated with an automated action program in Image-Pro Plus (version 6.0; Media Cybernetics, Inc., Rockville, MD, USA).

Statistical analysis. Statistical analysis was performed with one-way analysis of variance followed by a Newman-Keuls test using GraphPad Prism software (version 6.0; GraphPad Software Inc., La Jolla, CA, USA). The Spearman correlation test was conducted to analyze the existing associations. $\mathrm{P}<0.05$ was considered to indicate a statistically significant difference.

\section{Results}

Loss of FRCs in TDLNs. Lewis lung carcinoma cells were inoculated in the right inguinal region of 6-week-old C57BL/6 mice. Following tumor inoculation for 28 days, mice were sacrificed and LNs from the two sides of the inguinal region were collected. LNs were then digested thoroughly with enzyme mix comprised of RPMI-1640 containing collagenase IV and DNase I (24). Using flow cytometry, a significant decrease $(\mathrm{P}<0.01)$ was observed in the number of FRCs $\left(\mathrm{gp} 38^{+} \mathrm{CD} 31^{-}\right)$ in CD45 cells in TDLNs compared with the control group (Fig. 1A). ER-TR7 and gp38 were reported as markers of FRCs and required for proper formation and organization of LNs $(11,28,29)$. To validate the effect of tumors on stromal cells in LNs, immunofluorescent staining of ER-TR7 was performed, which demonstrated that the density of ER-TR7 ${ }^{+}$FRCs was markedly decreased in TDLNs compared with control LNs (Fig. 1B). Western blot analysis of gp38 showed a similar change in TDLN (Fig. 1C). These results indicated that the population of FRCs in TDLNs was affected in tumor-bearing mice. 
A
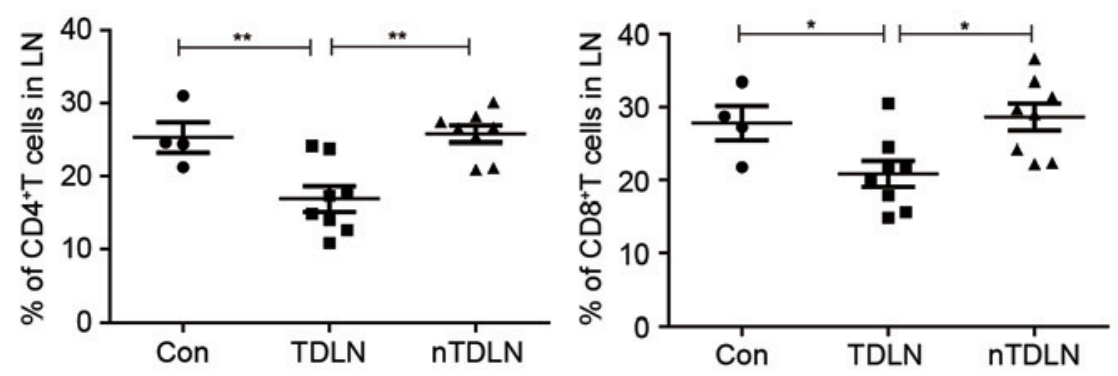

B
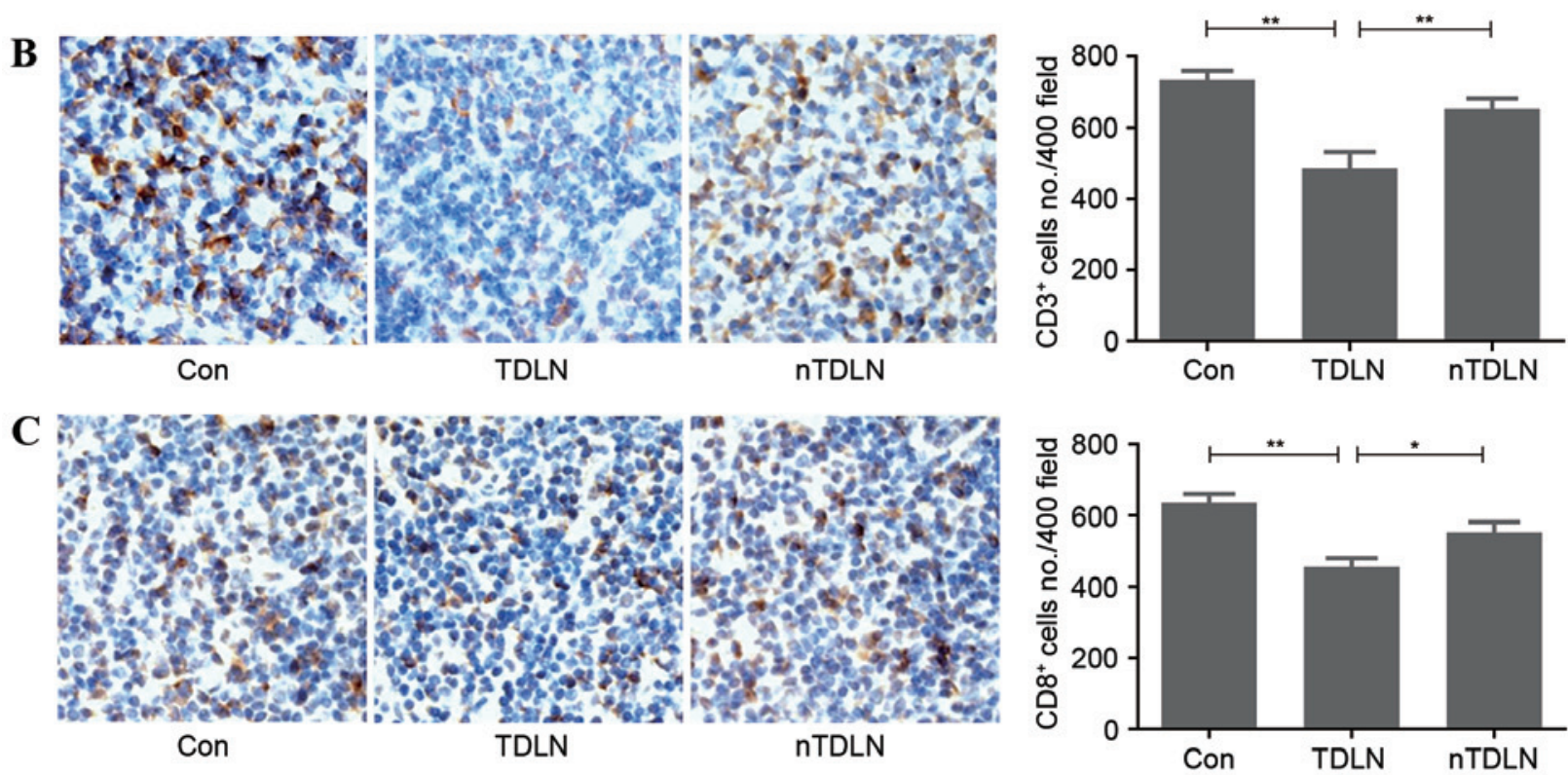

Figure 2. The number of T lymphocytes in TDLNs was reduced. (A) Flow cytometric analysis showed that the number of CD4 ${ }^{+}$and $\mathrm{CD}^{+} \mathrm{T}$ cells decreased significantly in TDLNs, representative of 4 mice for the control group and 8 mice for the tumor-bearing group. (B) LNs biopsies from mice were sectioned and stained for CD3. Staining was quantified by the number of cells with CD3 positivity. (C) LN biopsies from mice were sectioned and stained for CD8. Staining was quantified by the number of cells with $\mathrm{CD} 8$ positivity. The mean values \pm standard error of mean were analyzed from 3 mice per group. ${ }^{*}<0.05$, ${ }^{* *} \mathrm{P}<0.01$. LN, lymph node; TDLN, tumor draining lymph node; CD, cluster of differentiation; nTDLN, non tumor draining lymph node.

IL-7 is significantly decreased in TDLNs. FRCs are resident in LNs and generate the majority of IL-7 in the periphery, which is essential for the homeostasis of lymphocytes (10). It was examined whether loss of FRCs impacts IL-7 expression. IL-7 transcript measured by RT-qPCR was reproducibly decreased by more than one half in the TDLNs compared with contralateral non-TDLNs and control groups (Fig. 1D). Immunofluorescent staining of IL-7 confirmed the descent of IL-7 expression in TDLNs (Fig. 1E), as was gp38 detection in TDLNs compared with those of the control. These results indicated that loss of FRCs in TDLNs was responsible for the decreased IL-7 secretion.

The number of T lymphocytes is reduced in TDLNs. T cell homeostasis means that a relatively stable number of T lymphocytes in the periphery rely on IL-7 (10). They compete for this limited survival factor in LNs to maintain the T lymphocyte pool at a constant size (11). The present study aimed to identify whether the reduced level of IL-7 affects the number of total $\mathrm{T}$ cells in LNs. Flow cytometric analysis demonstrated that the number of $\mathrm{CD}^{+}$and $\mathrm{CD}^{+} \mathrm{T}$ cells decreased significantly in TDLNs (Fig. 2A). Immunohistochemistry results also confirmed that the amount of $\mathrm{CD}^{+} \mathrm{T}$ cells had markedly fallen in TDLNs (Fig. 2B). The number of $\mathrm{CD}^{+} \mathrm{T}$ cells was also calculated in the same way. In line with the downward trend of total number of $\mathrm{T}$ cells, $\mathrm{CD} 8^{+} \mathrm{T}$ cells decreased by $\sim 15 \%$ in TDLNs (Fig. 2C). Less T cells survived in TDLNs, indicating that less functional $\mathrm{T}$ cell may be armed to fight against the tumor burdens.'

TDLNs in patients with colon cancer. In the present study, a total of 40 patients diagnosed with colon cancer who had received surgical excision were selected. Each group contained 10 patients, according to their Dukes stage (A-D). There were 1 to $4 \mathrm{LNs}$ obtained from each patient. All LNs came from mesenteric LNs which were defined as TDLNs. Desmin was reported to be another marker of FRCs in LNs (28). Consequently, antibodies to desmin were used to label the FRC network in human LNs. Immunohistochemistry was performed to check the expression of IL-7 and desmin in human LNs. The expression area of desmin shrunk by up to $60 \%$, accompanied with tumor progression (Fig. 3A). The percentage of area that stained positive for IL-7 had a downward trend, and the differences between Dukes A and D stages showed statistical significance (Fig. 3B). With less IL-7 in TDLNs, a rapid and parallel decline of $\mathrm{CD}^{+}$ $\mathrm{T}$ cells was detectable in the Dukes B stage and accelerated markedly in the Dukes D stage (Fig. 3C).

Association between desmin, IL-7 and the number of $C D 8^{+}$ T cells. A significant positive association was identified 
A

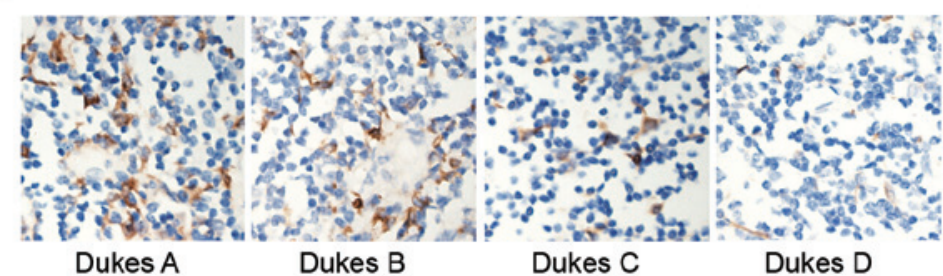

B

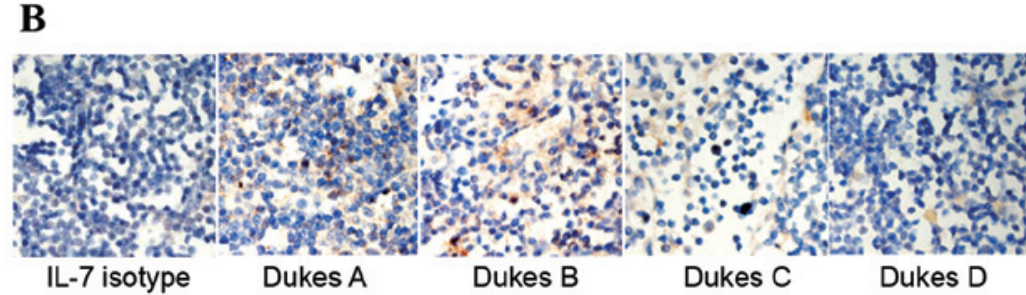

C

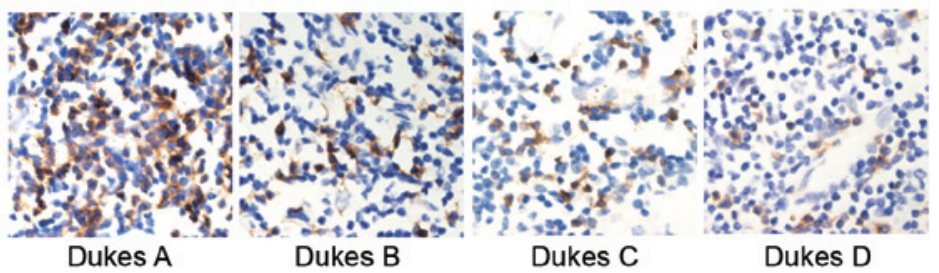

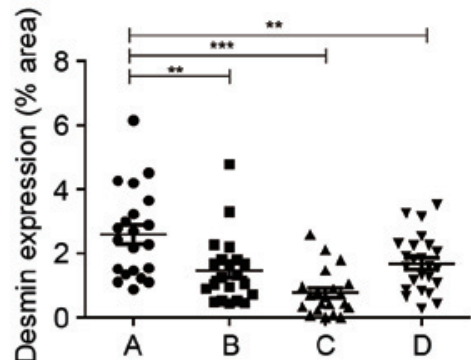
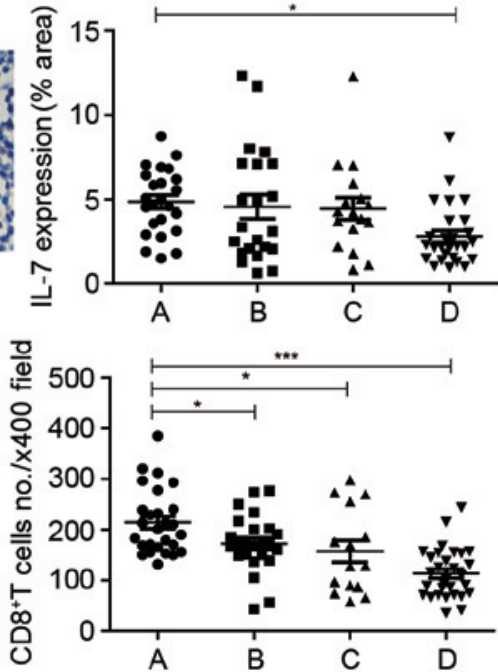

Figure 3. Desmin, IL-7 and CD8 ${ }^{+}$T cells decreased in human TDLNs. (A) LNs biopsies from patients with colon cancer were stained for desmin. QIA showed that the expression area of desmin shrunk by up to $60 \%$ accompanied with tumor progression. (B) LNs biopsies from patients with colon cancer were stained for IL-7. QIA showed that IL-7 is significantly reduced in Dukes D stage. (C) Representative sections stained for CD8 were shown from patients at different Dukes stages. QIA demonstrated that $\mathrm{CD}^{+} \mathrm{T}$ cells reduced with tumor progression. ${ }^{*} \mathrm{P}<0.05,{ }^{* *} \mathrm{P}<0.01 ;{ }^{* * *} \mathrm{P}<0.001$. IL-7, interleukin 7; LN, lymph node; TDLN, tumor draining lymph node; QIA, quantitative image analysis.
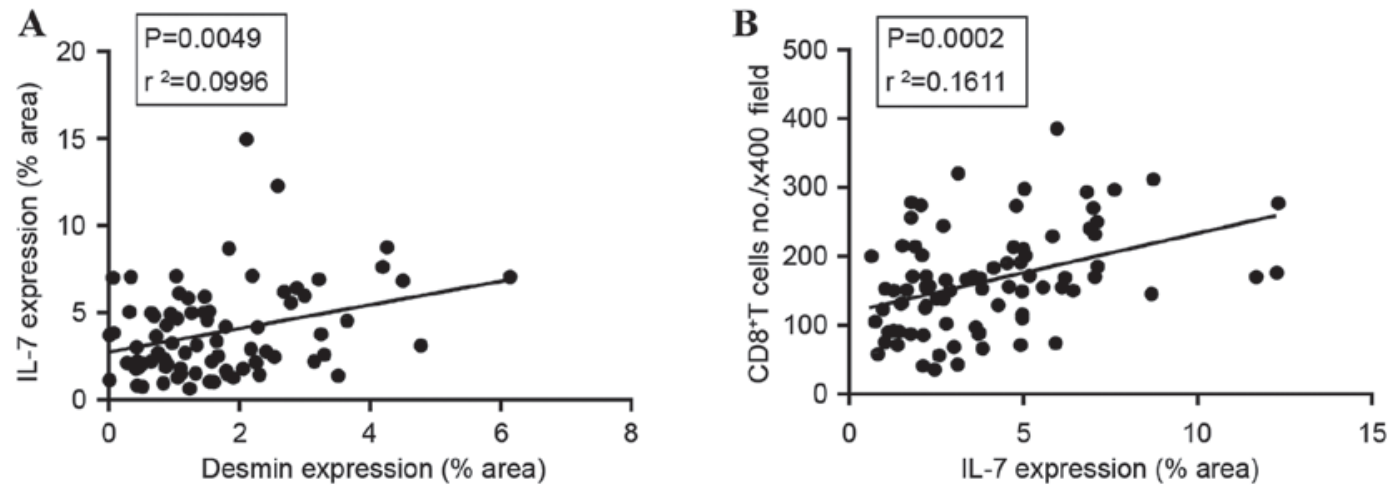

Figure 4. The Spearman correlation test was conducted to analyze the existing correlations. (A) Correlative analysis between desmin and IL-7 expression area were performed for all subjects. (B) Correlative analysis between IL-7 staining and the number of CD8 ${ }^{+} \mathrm{T}$ cells were performed for all subjects. IL-7, interleukin-7.

between desmin and IL-7 expression ( $\mathrm{P}=0.0049$; Fig. 4A). This coincided with the fact that FRCs were the main source of IL-7. The amount of $\mathrm{CD}^{+} \mathrm{T}$ cells was also significantly associated with IL-7 expression area ( $\mathrm{P}=0.0002$; Fig. 4B), since IL-7 was necessary for $\mathrm{CD}^{+} \mathrm{T}$ cell survival.

\section{Discussion}

Immunosuppression is a serious problem for patients with cancer (2). Tumor growth may result in $\mathrm{T}$ cell dysfunction by alterations in $\mathrm{T}$ cell receptor-cluster of differentiation 3 complex and induction of $\mathrm{T}$ cell tolerance (30). T cells also produce immunosuppressive cytokines, including transforming growth factor- $\beta 1$ (TGF- $\beta 1$ ) and granulocyte-macrophage colony-stimulating factor, to promote tumor escape from immunological surveillance $(31,32)$. LN draining of pathological sites is critical in immune function for the generation of adaptive immune responses. However, TDLNs are often detected with tumor invasion as early as it was first diagnosed.

FRCs in LNs orchestrate a complex network of extracellular matrix and collagens. They facilitate immune responses 
and maintain $\mathrm{T}$ cell homeostasis through the production of IL-7. Previous studies of pathogen infection in vivo have stated that a number of viruses, including LCMV and SIV, may infect the FRCs directly, accompanied with disruption of the conduit network $(11,33,34)$. Impaired FRC networks during human immunodeficiency virus infection accounted for increased apoptosis and loss of naïve T cells (35). A mouse tumor model of melanomas also indicated alterations of the stromal cell network only in inflammatory TDLNs, which profoundly altered the distribution of $\mathrm{T}$ lymphocytes as a result (36). The present study demonstrated that different tumor cells may influence the number of FRCs in TDLNs. Loss of FRC networks reduced the major source of IL-7 and decreased access to IL-7 (35). Consequently, there were less T lymphocytes left in the TDLNs. This may partly explain the weakened ability of immune surveillance of TDLNs.

Tumor cells may secrete certain types of soluble factors that directly impede immune reactions (36). In regard to the way in which tumors may affect FRCs, previous studies have detected higher TGF- $\beta$ mRNA expression in TDLNs (37). TGF- $\beta$ is reported to downregulate stromal IL-7 secretion in vitro (38). Otherwise, the association between tumor and stromal cells in LNs requires additional study.

In conclusion, the present results indicated that tumor cells may directly affect TDLNs by decreasing the FRC population, leading to less IL-7 secretion. As a result, the amount of T cells in TDLNs declined with less survival signals. This may have partly contributed to the explanation of cancer immunosuppressive theories.

\section{Acknowledgements}

The authors thank the Department of Pathology, Xinqiao Hospital (Chongqing, China), for providing LN biopsy specimens of patients, and for performing paraffin sections. The present study was supported by the National Nature Science Foundation of China (grant nos. 81472648 and 81500089), the outstanding Youth Scientist Foundation of Chongqing (grant nos. CSTC and 2008BA5035) and the National Key Basic Research Program of China (973 program; grant nos. 010CB529404 and 2012CB526603).

\section{References}

1. Hanahan D and Weinberg RA: Hallmarks of cancer: The next generation. Cell 144: 646-674, 2011.

2. Gao J, Zhao L, Wan YY and Zhu B: Mechanism of action of IL-7 and its potential applications and limitations in cancer immunotherapy. Int J Mol Sci 16: 10267-10280, 2015.

3. Lindau D, Gielen P, Kroesen M, Wesseling P and Adema GJ: The immunosuppressive tumour network: Myeloid-derived suppressor cells, regulatory $\mathrm{T}$ cells and natural killer T cells. Immunology 138: 105-115, 2013.

4. Malhotra D, Fletcher AL and Turley SJ: Stromal and hematopoietic cells in secondary lymphoid organs: Partners in immunity. Immunol Rev 251: 160-176, 2013.

5. Munn DH and Mellor AL: The tumor-draining lymph node as an immune-privileged site. Immunol Rev 213: 146-158, 2006.

6. Turley SJ, Fletcher AL and Elpek KG: The stromal and haematopoietic antigen-presenting cells that reside in secondary lymphoid organs. Nat Rev Immunol 10: 813-825, 2010.

7. Bajenoff M, Egen JG, Koo LY, Laugier JP, Brau F, Glaichenhaus N and Germain RN: Stromal cell networks regulate lymphocyte entry, migration, and territoriality in lymph nodes. Immunity 25: 989-1001, 2006.
8. Zhao L, Gao J, Li Y, Liu L, Yang Y, Guo B and Zhu B: Disrupted homeostatic cytokines expression in secondary lymph organs during HIV infection. Int J Mol Sci 17: 413, 2016.

9. Zhao L, Chen J, Liu L, Gao J, Guo B and Zhu B: Essential role of TNF-alpha in development of spleen fibroblastic reticular cells. Cell Immunol 293: 130-136, 2015.

10. Link A, Vogt TK, Favre S, Britschgi MR, Acha-Orbea H, Hinz B, Cyster JG and Luther SA: Fibroblastic reticular cells in lymph nodes regulate the homeostasis of naive T cells. Nat Immunol 8: 1255-1265, 2007.

11. Mueller SN and Germain RN: Stromal cell contributions to the homeostasis and functionality of the immune system. Nat Rev Immunol 9: 618-629, 2009.

12. Onder L, Narang P, Scandella E, Chai Q, Iolyeva M, Hoorweg K, Halin C, Richie E, Kaye P, Westermann J, et al: IL-7-producing stromal cells are critical for lymph node remodeling. Blood 120: 4675-4683, 2012.

13. Fletcher AL, Malhotra D and Turley SJ: Lymph node stroma broaden the peripheral tolerance paradigm. Trends Immunol 32: $12-18,2011$.

14. Lee JW, Epardaud M, Sun J, Becker JE, Cheng AC, Yonekura AR, Heath JK and Turley SJ: Peripheral antigen display by lymph node stroma promotes $\mathrm{T}$ cell tolerance to intestinal self. Nat Immunol 8: 181-190, 2007.

15. Dubrot J, Duraes FV, Potin L, Capotosti F, Brighouse D, Suter T, LeibundGut-Landmann S, Garbi N, Reith W, Swartz MA and Hugues S: Lymph node stromal cells acquire peptide-MHCII complexes from dendritic cells and induce antigen-specific CD4 ${ }^{+}$ T cell tolerance. J Exp Med 211: 1153-1166, 2014.

16. Kittipatarin $C$ and Khaled AR: Interlinking interleukin-7. Cytokine 39: 75-83, 2007.

17. Surh CD and Sprent J: Homeostasis of naive and memory T cells. Immunity 29: 848-862, 2008

18. Rathmell JC, Farkash EA, Gao W and Thompson CB: IL-7 enhances the survival and maintains the size of naive $\mathrm{T}$ cells. J Immunol 167: 6869-6876, 2001.

19. Fry TJ and Mackall CL: The many faces of IL-7: From lymphopoiesis to peripheral $\mathrm{T}$ cell maintenance. J Immunol 174: 6571-6576, 2005

20. Chappaz S and Finke D: The IL-7 signaling pathway regulates lymph node development independent of peripheral lymphocytes. J Immunol 184: 3562-3569, 2010.

21. Chappaz S, Gärtner C, Rodewald HR and Finke D: Kit ligand and Il7 differentially regulate Peyer's patch and lymph node development. J Immunol 185: 3514-3519, 2010.

22. Schmutz S, Bosco N, Chappaz S, Boyman O, Acha-Orbea H, Ceredig R, Rolink AG and Finke D: Cutting edge: IL-7 regulates the peripheral pool of adult ROR gamma+ lymphoid tissue inducer cells. J Immunol 183: 2217-2221, 2009.

23. Dukes CE: The surgical pathology of rectal cancer: President's address. Proc R Soc Med 37: 131-144, 1944.

24. Fletcher AL, Malhotra D, Acton SE, Lukacs-Kornek V, Bellemare-Pelletier A, Curry M, Armant M and Turley SJ: Reproducible isolation of lymph node stromal cells reveals site-dependent differences in fibroblastic reticular cells. Front Immunol 2: 35, 2011.

25. Sawa Y, Arima Y, Ogura H, Kitabayashi C, Jiang JJ, Fukushima T, Kamimura D, Hirano T and Murakami M: Hepatic interleukin-7 expression regulates T cell responses. Immunity 30: 447-457, 2009.

26. Livak KJ and Schmittgen TD: Analysis of relative gene expression data using real-time quantitative PCR and the 2(-Delta Delta C(T)) method. Methods 25: 402-408, 2001.

27. Zeng M, Paiardini M, Engram JC, Beilman GJ, Chipman JG, Schacker TW, Silvestri G and Haase AT: Critical role of CD4 T cells in maintaining lymphoid tissue structure for immune cell homeostasis and reconstitution. Blood 120: 1856-1867, 2012.

28. Zeng M, Smith AJ, Wietgrefe SW, Southern PJ, Schacker TW, Reilly CS, Estes JD, Burton GF, Silvestri G, Lifson JD, et al: Cumulative mechanisms of lymphoid tissue fibrosis and $\mathrm{T}$ cell depletion in HIV-1 and SIV infections. J Clin Invest 121: 998-1008, 2011.

29. Astarita JL, Acton SE and Turley SJ: Podoplanin: Emerging functions in development, the immune system, and cancer. Front Immunol 3: 283, 2012.

30. de Aquino MT, Malhotra A, Mishra MK and Shanker A: Challenges and future perspectives of $\mathrm{T}$ cell immunotherapy in cancer. Immunol Lett 166: 117-133, 2015.

31. Gorelik L and Flavell RA: Immune-mediated eradication of tumors through the blockade of transforming growth factor-beta signaling in T cells. Nat Med 7: 1118-1122, 2001. 
32. Wang Y, Han G, Wang K, Liu G, Wang R, Xiao H, Li X, Hou C, Shen B, Guo R, et al: Tumor-derived GM-CSF promotes inflammatory colon carcinogenesis via stimulating epithelial release of VEGF. Cancer Res 74: 716-726, 2014.

33. Mueller SN, Matloubian M, Clemens DM, Sharpe AH, Freeman GJ, Gangappa S, Larsen CP and Ahmed R: Viral targeting of fibroblastic reticular cells contributes to immunosuppression and persistence during chronic infection. Proc Natl Acad Sci USA 104: 15430-15435, 2007.

34. Choi YK, Fallert BA, Murphey-Corb MA and Reinhart TA: Simian immunodeficiency virus dramatically alters expression of homeostatic chemokines and dendritic cell markers during infection in vivo. Blood 101: 1684-1691, 2003.

35. Zeng M, Haase AT and Schacker TW: Lymphoid tissue structure and HIV-1 infection: Life or death for T cells. Trends Immunol 33 . 306-314, 2012.
36. Soudja SM, Henri S, Mello M, Chasson L, Mas A, Wehbe M, Auphan-Anezin N, Leserman L, Van den Eynde B and SchmittVerhulst AM: Disrupted lymph node and splenic stroma in mice with induced inflammatory melanomas is associated with impaired recruitment of $\mathrm{T}$ and dendritic cells. PloS One 6: e22639, 2011.

37. Imai K, Minamiya $\mathrm{Y}$, Koyota S, Ito M, Saito H, Sato Y, Motoyama S, Sugiyama T and Ogawa J: Inhibition of dendritic cell migration by transforming growth factor- $\beta 1$ increases tumor-draining lymph node metastasis. J Exp Clin Cancer Res 31: 3, 2012.

38. Tang J, Nuccie BL, Ritterman I, Liesveld JL, Abboud CN and Ryan DH: TGF-beta down-regulates stromal IL-7 secretion and inhibits proliferation of human B cell precursors. J Immunol 159: 117-125, 1997. 